\title{
Measurements of Intermittency in the Upper Stratosphere and Mesosphere
}

\author{
R. J . Sica and M. D. Thorsley \\ Department of Physics \\ The University of Western Ontario \\ London, Ontario N6A 3K 7 \\ Canada
}

\section{Introduction}

Measurements of the gravity wave spectrum in the upper stratosphere and mesosphere are scarce compared to those in the troposphere and lower thermosphere, which are easier to probe via in situ and remote sensing techniques. This paucity of measurements impacts the parameterization of gravity waves in general circulation models of the middle atmosphere, since the effects of gravity waves are not adequately constrained by measurements. The development of a larger power-aperture product lidar at The University of Western Ontario has allowed the measurement of the gravity wave spectrum at high temporal-spatial resolution. These measurements have been used to impact three key areas relevant to parameterizations: the thermodynamic perturbations caused by gravity waves, the variability of the spatial and temporal spectra and the estimation of the eddy diffusion coefficient. After a brief introduction to the instrumentation (Section 2), Section 3 describes an extension of the initial measurements of superadiabatic lapse rates by Sica and Thorsley (1996a). A taste of the spatial and temporal spectral retrievals possible with the lidar measurements is given in Section 4. In 
Section 5, the spectra are used to cal culate energy dissipation and the eddy diffusion coefficient.

\section{Instrumentation}

The Purple Crow Lidar is a monostatic lidar system which can simultaneously measure both Rayleigh and sodium resonance fluorescence backscatter. The lidar is located just outsi de of London, Ontario, Canada at the Delaware Observatory ( $\left.42^{\circ} 52^{\prime} \mathrm{N} ; 81^{\circ} 23^{\prime} \mathrm{W}\right)$. The Purple Crow Lidar enjoys a large power-aperture product by the use of a high power transmitter and large aperture receiver. The transmitter is a Nd:YAG laser operating at the second harmonic, with a output energy of nominally $600 \mathrm{~mJ} /$ pulse at $20 \mathrm{~Hz}$. The receiver is a $2.65-\mathrm{m}$ diameter liquid mercury mirror, which is coupled to a photon counting system by a optical fiber. Sica et al. (1995) show that the liquid mirror has a performance similar to a traditional glass telescope of the same area. The measurements are obtained every $1 \mathrm{~min}$ in $24 \mathrm{~m}$ range bins. Temperatures are found from the density profiles as described by Chanin and Hauchecorne (1984). The density fluctuations are determined by the methodology of Gardner et al. (1989b).

\section{Measurements of Superadiabatic Lapse Rates}

The methodol ogy for determining layers of stability and potential instability is, by necessity, tedious (Sica and Thorsley 1996a). In principle one could simply take a temperature profile, differentiate it and be done. In practice, despite the large return photocount signal, the temperature 
changes sought are extremely small. Furthermore, the expectation is, that higher in the atmosphere more unstable regions will exist, as the amplitude of the waves increases with height. Unfortunately, the lidar return signal decreases for the same reason, and in fact, faster than the gravity waves grow due to the exponential decrease of density with height. Hence, extreme care must be taken in the determination of stable and potentially unstable regions.

The photon noise in the temperature profiles, if sufficiently large, can cause the appearance of superadiabatic layers in an otherwise stable temperature profile. To help determine if the lapse rates measured are due solely to noise, a synthetic data set for each night is made, or "cloned", using the average density profile from the measurements for the shape of the photocount profile. Gaussian noise is added and lapse rates are calculated after which each individual measurement and cloned data point (and their associated error) is then treated as the mean and variance of a Gaussian distribution. Distributions in which $80 \%$ of the probability lies bel ow zero are classified as negative and vice versa for positive. Distributions which fall in neither category are indeterminate. With the differences in lapse rates and their signs available for both the measurements and the cloned data, the two distributions can be statistically tested to determine whether or not they are likely to arise from the same parent population. Kupier's variant of the Kolmogorov-Smirnov test is used to test whether or not the measurements and the cloned data are from the same distribution, while the F-test is used to test for significantly different variances between the data sets (Press et al. 1992). 
On some nights the variance of the lapse rate measurements is significantly different from the photocount noise. The difference between these variances is a measure of atmospheric variability. To illustrate this variability, a representative grouping of nights from the Purple Crow Lidar's database is sampled (Table 1). The photon noise on all these

Table 1. Four groups used to study the occurrence of positive and negative layers. The group name is in the first row, while subsequent cells contain the nights in the group (year-month-day format).

\begin{tabular}{cccc}
\hline \hline Oct94 & J un95 & Oct95 & May96 \\
\hline 94/10/7 & $95 / 6 / 1$ & $95 / 10 / 12$ & $96 / 5 / 13$ \\
$94 / 10 / 12$ & $95 / 6 / 13$ & $95 / 10 / 13$ & $96 / 5 / 31$ \\
$94 / 10 / 23$ & $94 / 6 / 15$ & $95 / 10 / 17$ & $96 / 6 / 1$ \\
$94 / 10 / 24$ & & & \\
\hline
\end{tabular}

nights is similar. The atmospheric variability calculated for each group is shown in Figure 1. The Oct94 group has essentially no variability above $40 \mathrm{~km}$, as the photon noise dominates. However, the other groups

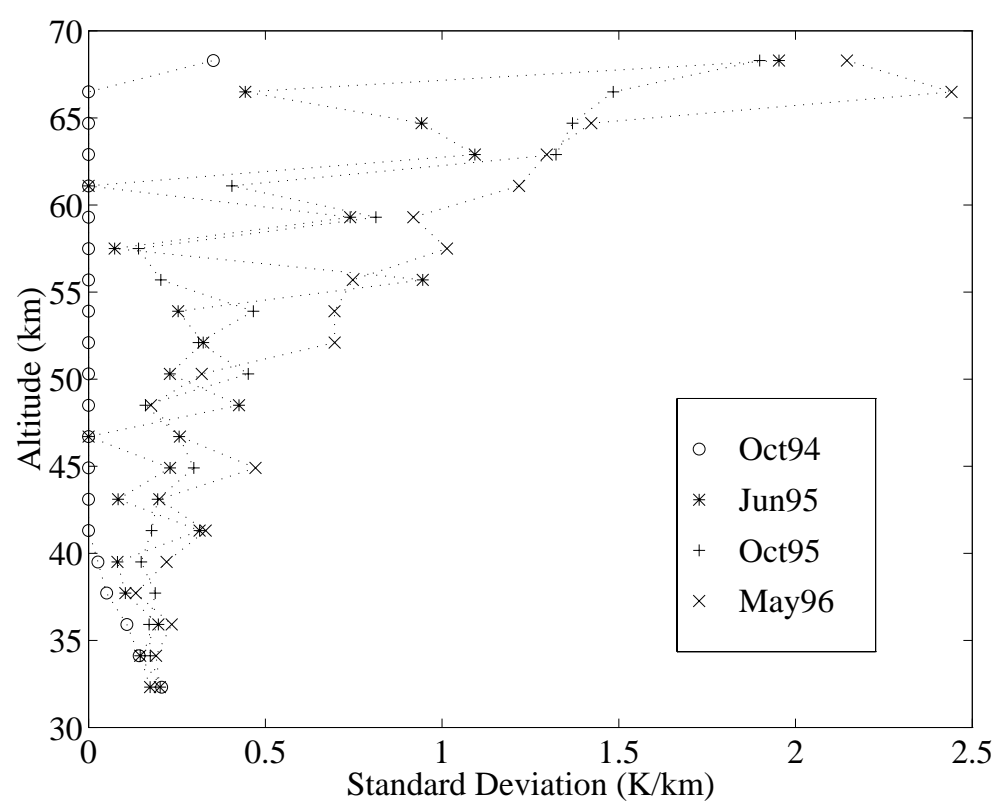

Fig. 1. The difference between the standard deviations of the lapse rate measurements and the photocount noise for the four groups. 
all have residual variability. To further illustrate the difference between the Oct94 group and the other groups, the difference between the percentage of space and time with positive, negative and indeterminate lapse rate differences can be compared. The percent differences in time are shown in Figure 2. The number of negative regions is between
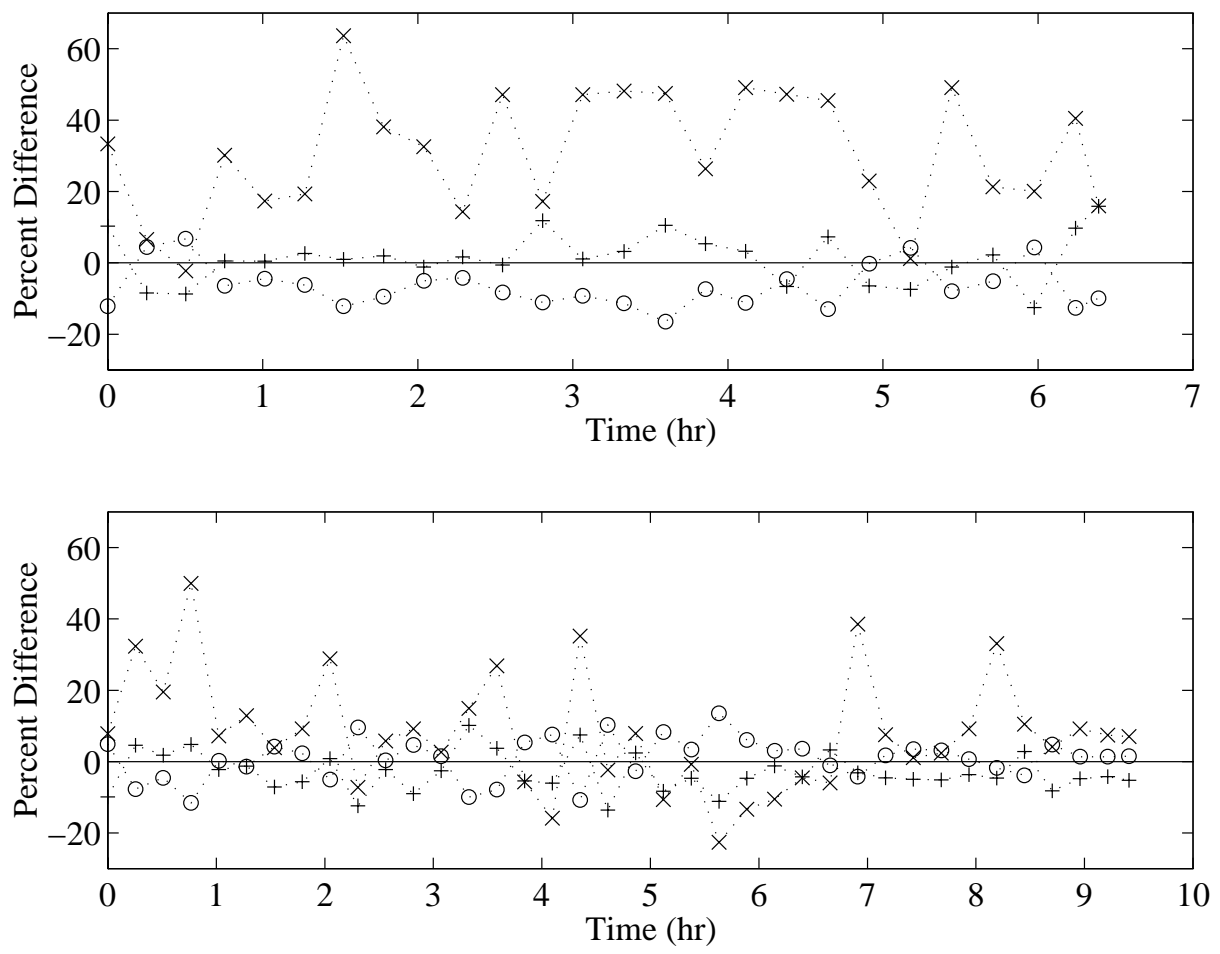

Fig. 2. Percent difference in time between the lapse rate measurements and synthetic data for the variable pod (top) and the noise pod (bottom) for positive (' + '), negative (' $x$ ') and indeterminate (' $O$ ') differences.

$20-30 \%$ greater for the average of the J un95, Oct95 and May96 groups (henceforth the "variable" pod) compared to the cloned data, while the indeterminate percent differences are about 10\% less. The Oct94 group (henceforth the "noise" pod) has essentially the same number of positive, negative and indeterminate layers as the cloned data. The spatial percent differences are shown in Figure 3. In the stratosphere, bel ow 40 $\mathrm{km}$ both pods show large differences from the cloned data. In the mesos- 



Fig. 3. Percent difference in space between the lapse rate measurements and synthetic data for the variable pod (left) and the noise pod (right) for positive (' + '), negative (' $x$ ') and indeterminate (' $O$ ') differences. The height ranges have been plotted on difference scales for clarity.

phere, the variable pod percent differences are consistent with a distribution with a larger variance than the photocount noise. The residual attributed to atmospheric variability (Fig. 1 ) is consistent with the comparison between the percent differences.

The spatial percentages of positive, negative and indeterminate layers are shown in Figure 4. Throughout most of the upper stratosphere the positive regions dominate, while the increase of the negative regions is roughly monotonically increasing with height. Above the stratopause, the number of indeterminate regions is al most al ways a few percent less than $60 \%$, as compared to an average of about $63 \%$ for the indeterminate layers in the noise pod. 


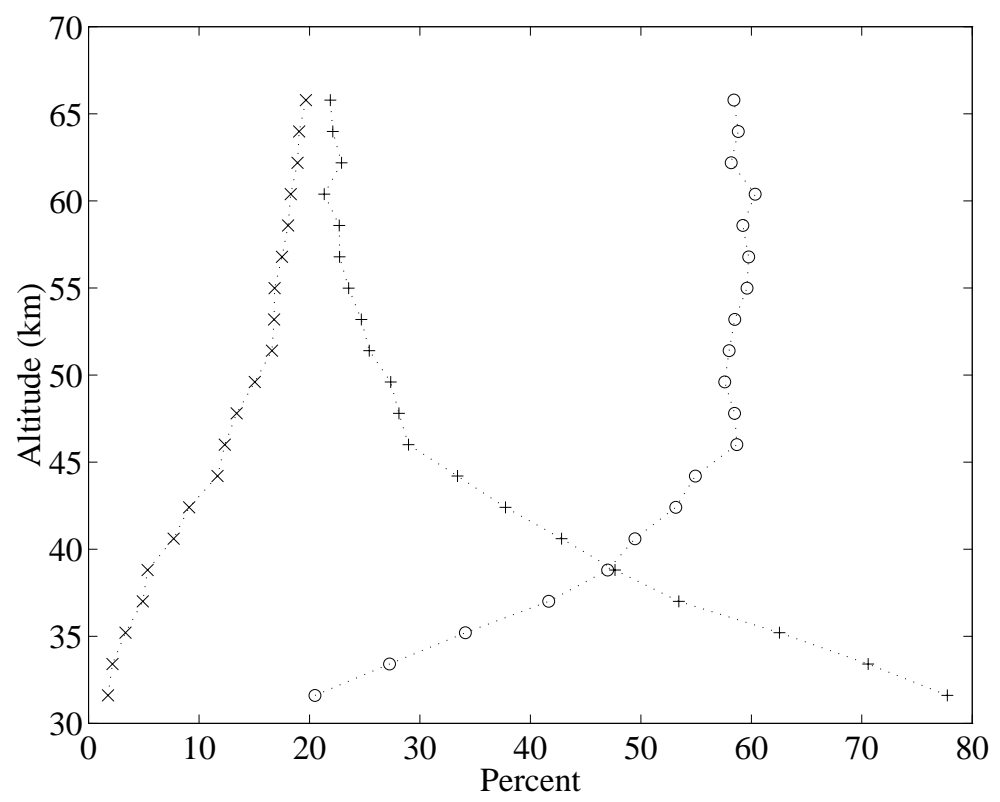

Fig. 4. Percentage of positive (' + '), negative (' $x$ ') and indeterminate regions ('o') as a function of height for the variable pod.

Hamilton (1984) computed the Richardson number for a large body of sounding rocket measurements. The nearest station to London, Ontario in his study is Wallops I sland, Virginia, about $5^{\circ}$ of Iatitude to the south. The percentage of regions with $\mathrm{Ri}<1$ for Hamilton's averages during equinox agrees well in the stratosphere with the negative percentages, but are about $40 \%$ lower in the mesosphere.

The coherence of the variable pod can be found in the manner described by Sica and Thorsley (1996a). The coherence, that is the distribution of contiguous positive, negative and indeterminate regions in space or time, is shown in Figure 5. The general distributions are similar to those on an individual night. The negative regions are extremely confined in space and time relative to the positive regions.

The individual lapse rate differences can be plotted as a function of height and time. In the mesosphere, these plots are similar from night to night. However, below $40 \mathrm{~km}$, substantial variations in the amount and duration of layers is evident on different nights. Figure 6 shows the 



Fig. 5. Spatial (left) and temporal (right) coherence for the variable pod. The numbers in brackets are the number of measurements in each distribution

lapse rate differences on October 12, 1995. Below 40 km, substantial positive regions exist (e.g. from 0200 - 0300 hr; 0400-0500 hr, etc.). On

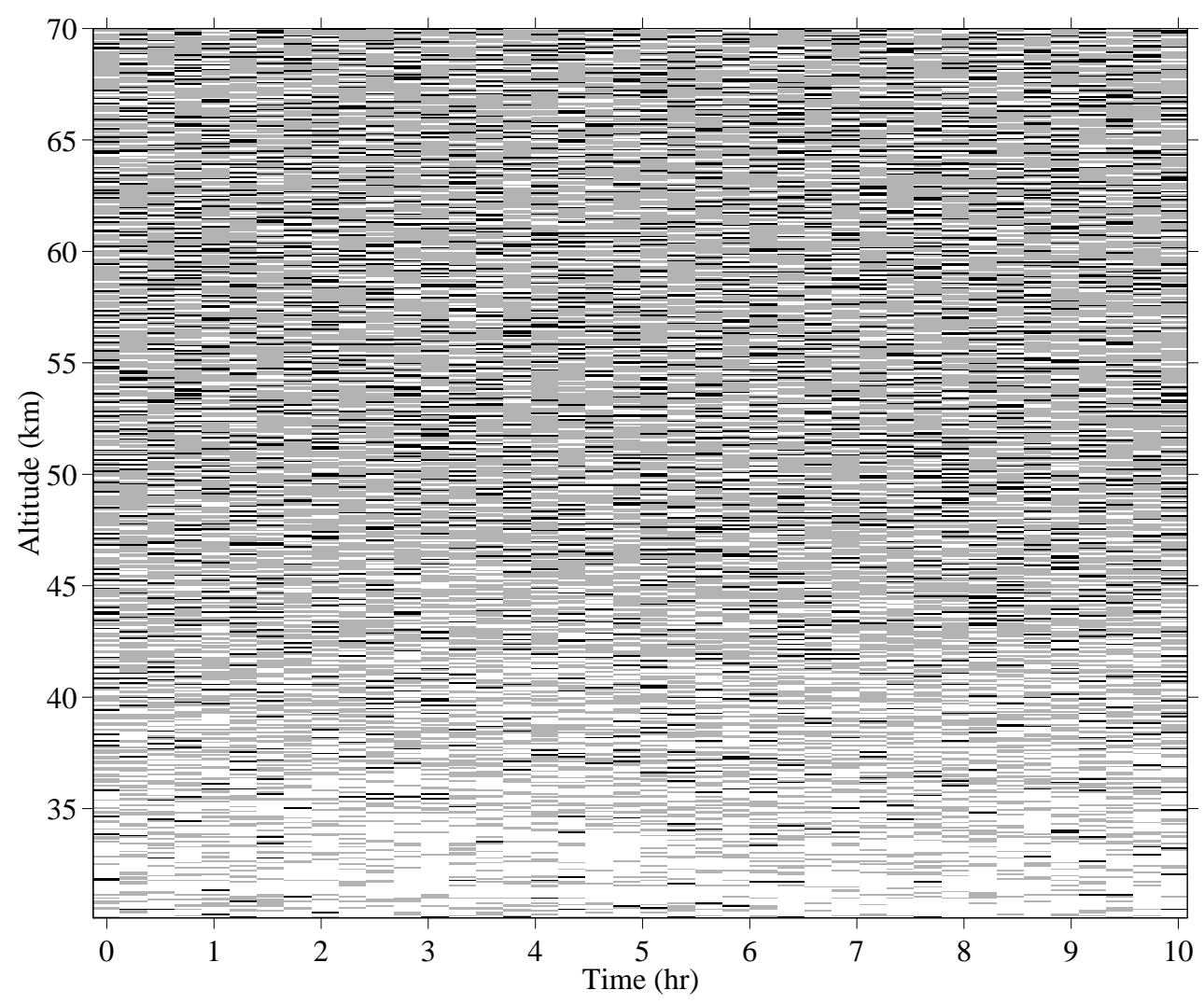

Fig. 6. Individual lapse rate differences on October 12, 1995. Positive regions are white, indeterminate regions are gray and negative regions black. Zero time on the ordinate is 2024 E DT. 
October 17, 1995 few extended regions of stability exist compared to October 12 (Fig. 7). There are also substantially more negative layers below $40 \mathrm{~km}$ on this night.

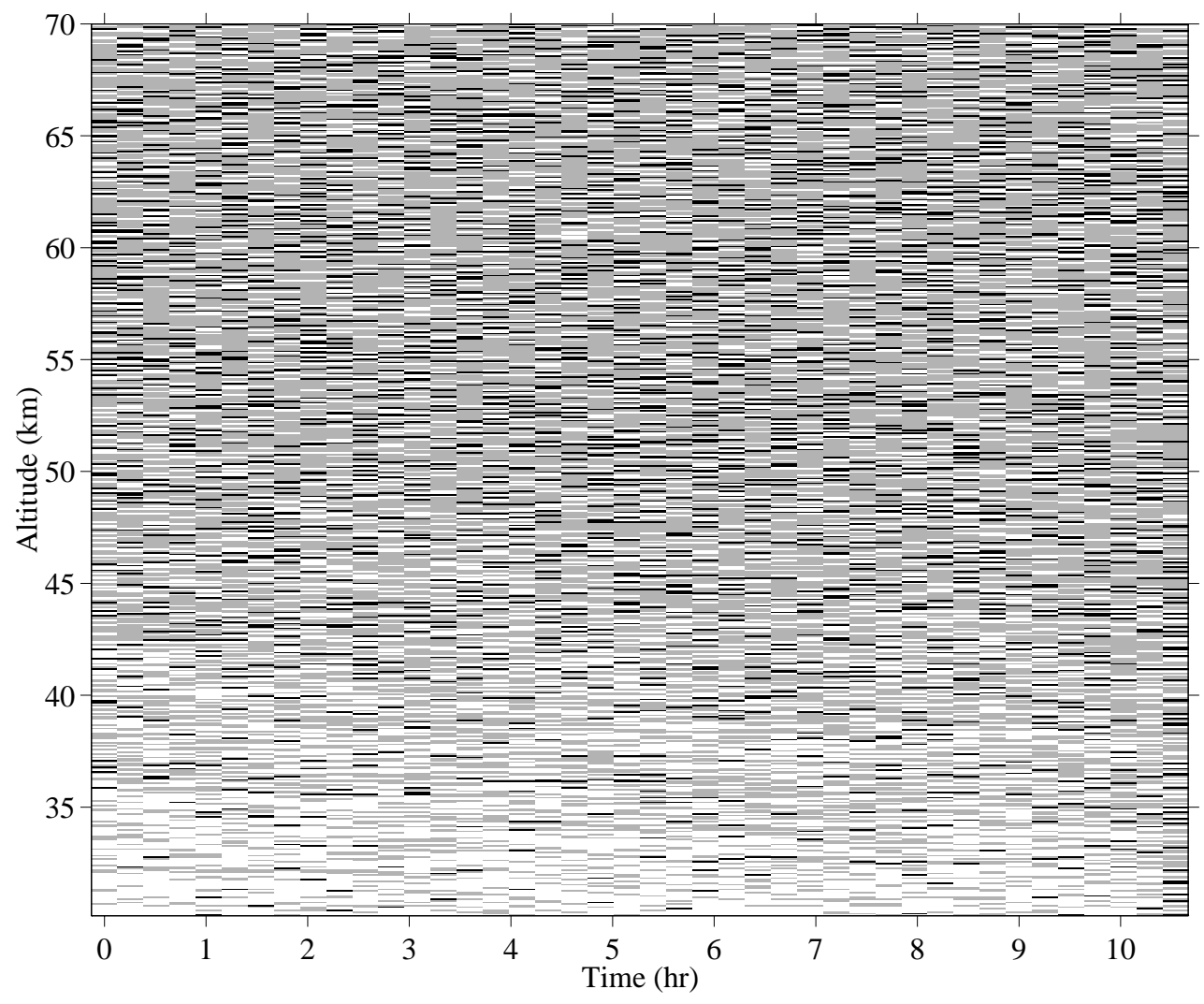

Fig. 7. Individual lapse rate differences on October 17, 1995. Positive regions are white, indeterminate regions are gray and negative regions black. Zero time on the ordinate is 2009 EDT.

\section{Vertical Wavenumber and Temporal Spectrum}

The material in this and the following section summarizes just a few of the main points reached in a detailed study by Sica (1996b; 1996c). The measurements used for this study were obtained on the night of August 30, 1994 commencing at 2319 EDT. The vertical (temporal) density fluctuations are temporally (spatially) filtered using finite impulse response filters. The data series were smoothed using either a Kaiser-Bessel filter 
with $40 \mathrm{~dB}$ of attenuation (in space) or by 3s and 5s (in time; Hamming 1977). The power spectral densities calculated have units of meters (spatial) or seconds (temporal); that is the angular units have been removed. From these natural units the gravity wave polarization equations can be used to scale the spectrum to a kinetic energy density or velocity spectrum (Gardner et al. 1989a).

Spectral analysis of the density fluctuations was performed using parametric models. Statistical procedures such as correlogram analysis require the autocorrelation sequence of a process to be set to zero outside a specified spectral window. Parametric models can use information outside of this range, which eliminates the sidel obes associated with window functions and increases spectral resolution. Specific models of use for gravity wave spectral studies are the autoregressive model and the Multiple SI gnal Classification (MUSIC) frequency estimator (Marple 1987).

The average vertical wavenumber spectrum using the MUSIC algorithm is shown in Figure 8. The arrow on the ordinate indicates the Hines parameter (Hines 1991). The spectrum in the regions of and to the right of the arrow are considered to be nonlinear. The conversion from density fluctuations to kinetic energy density should be treated with caution in these regions. For the average vertical wavenumber spectrum in the upper stratosphere on this night the majority of the tail region is linear (that is to the left of the arrow).

The average temporal spectrum in this height range shows a monotonic descent, without the shoulder often seen in correlograms (Fig. 8). A strong feature is present at $2.2 \times 10^{-3} \mathrm{~s}^{-1}$. Closer analysis of the spectrum shows companion features to this strong feature at the second and 

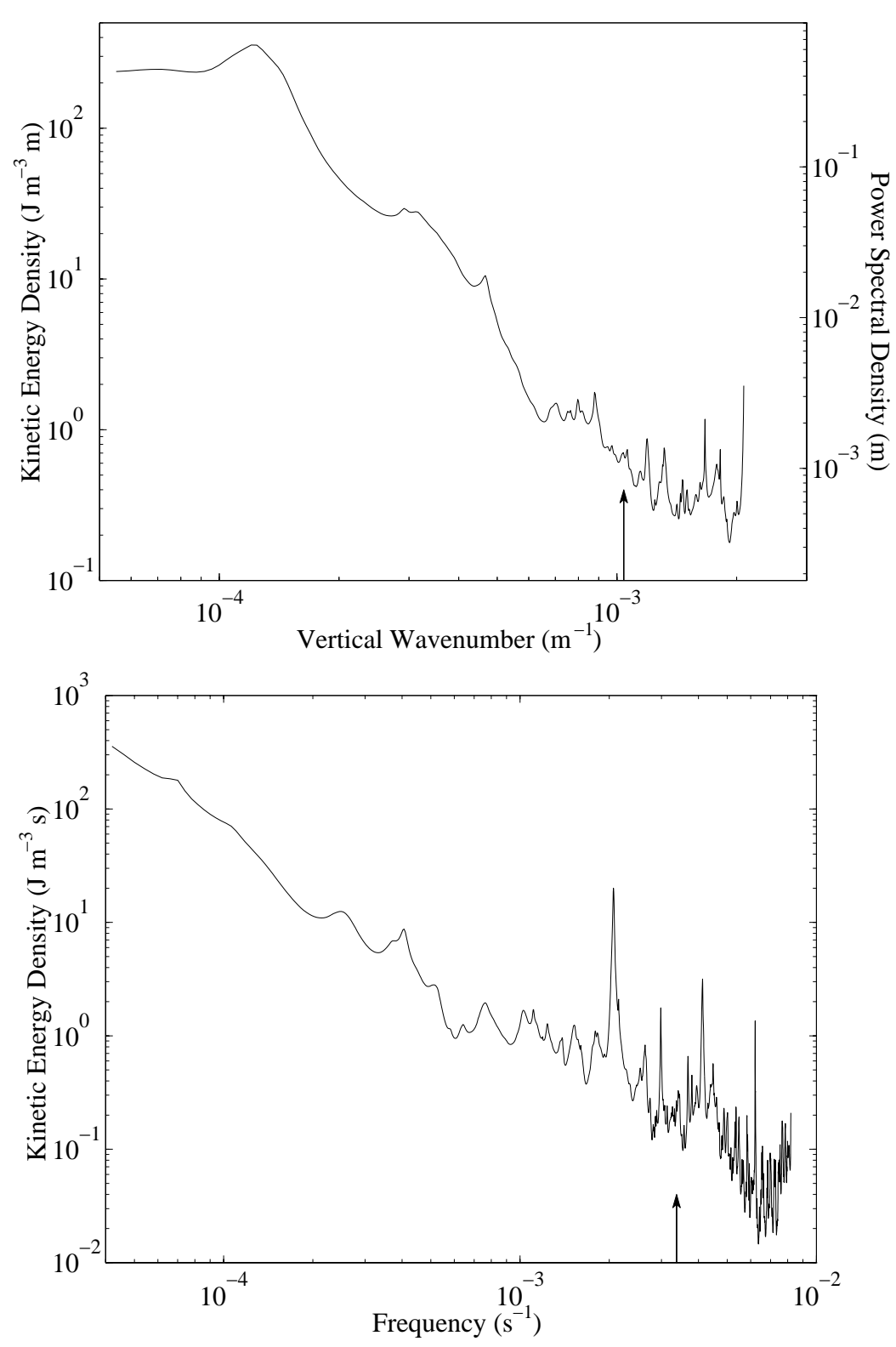

Fig. 8. Average vertical wavenumber (top) and temporal (bottom) spectrum in the upper stratosphere on August 30, 1994. The arrow on the vertical wavenumber spectrum indicates the Hines parameter, while the arrow on the temporal spectrum indicates the measured buoyancy frequency.

third harmonics. A detailed study of this event is in progress. The monotonic decrease of the spectrum continues down to the measured buoyancy frequency $\left(3.4 \times 10^{-3} \mathrm{~s}^{-1}\right)$. Below this point additional energy appears to be present, before the spectral energy rapidly decreases above $7 \times 10^{-3} \mathrm{~s}^{-1}$. 
The signal-to-noise ratio of the measurements coupled with the MUSIC algorithm allow the evolution of the spectrum with time to be examined. The individual one minute vertical wavenumber spectra can be shown as a function of time and wavenumber (Fig. 9). Note the coherence of

Fig. 9. Contour "image" for the individual MUSIC spectra in the upper stratosphere on the night of August 30, 1994. The ordinate is time while the abscissa is the logarithm of the kinetic energy density. The individual contours are separated by $10 \mathrm{~dB}$, with each gray-scale variation corresponding to a $10 \mathrm{db}$ decrease in kinetic energy density (white $0 \mathrm{~dB}$, black $-60 \mathrm{~dB}$ ). The peak value is $2950 \mathrm{~J} /$ $\mathrm{m}^{3} \mathbf{m}$. Zero hours corresponds to 2319 EDT.

many features well beyond the bandwidth of the temporal filtering ( 7 $\mathrm{min})$. At small wavenumbers, periods of high power exist between 0000 to $0100 \mathrm{hr}$ and 0230 to $0415 \mathrm{hr}$, while the peak power decreases -20 to 
$-30 \mathrm{~dB}$ at times during the interim period. During this time, the regions of highest power shift to higher wavenumbers (e.g. the period around $0200 \mathrm{hr}$ at $\left.-3.8=\log _{10}[1 /(6310 \mathrm{~m})]\right)$. The power in the middle wavenumber bands shifts to lower wavenumbers at $0200 \mathrm{hr}$, just prior to an enhancement of the power around $1 \times 10^{-4} \mathrm{~m}^{-1}$. At the highest wavenumbers several "islands" of power enhancement occur in the $1 \times 10^{-3}$ $\mathrm{m}^{-1}$ region. Some of these events (e.g. at $\left.-3.2=\log _{10}[1 /(1580 \mathrm{~m})]\right]$ and $0450 \mathrm{hr}$ ) are extremely intense, with peaks as high20 dB above the typical background.

\section{Calculation of Energy Dissipation and the Eddy Diffusion Coefficient}

The energy dissipation can be found from the product of the root mean square velocity and the standard deviation of the time rate of change of the velocity (Sica 1996c). The energy dissipation, which varies from a minimum of $1.5 \mathrm{~mW} / \mathrm{kg}$ to $80 \mathrm{~mW} / \mathrm{kg}$ near the stratopause, has a mean value of $11 \mathrm{~mW} / \mathrm{kg}$. The energy dissipation can also be calculated for individual waves by Prony's method, which allows the frequency, amplitude, growth rate and phase to be estimated from the measurements (Marple 1987). Once a fit to the data series has been computed, specific frequencies, if and when they exist, can be tracked as a function of time and the phase velocity, which is assumed equal to the group velocity determined. Knowing the group velocity, the growth rate and the amplitude of the wave perturbation expressed as an average horizontal wind fluctuation, the energy dissipation can be calculated without assuming a form for the spectrum (Hines 1965). A wave with a wavenumber of about $1 \times 10^{-4} \mathrm{~m}^{-1}$ and a monotonically decreasing phase is present from 
0000 to $0040 \mathrm{hr}$. The energy dissipation estimated from Hines' formula is about $70 \mathrm{~mW} / \mathrm{kg}$, consistent with the spectral calculation.

The energy dissipation can be used to estimate the eddy diffusion coefficient. The eddy diffusion coefficient, D, is proportional to the energy dissipation and inversely proportional to the square of the angular buoyancy frequency:

$$
D=\beta \frac{\varepsilon}{N^{2}}
$$

where $\beta$ is traditionally taken as a constant. Hocking (1991) discusses the choices of $\beta$ typically employed, with values in the range between 0.2 and 1.0. However, it has recently been pointed out by Mclntyre (1989) that $\beta$ actually varies as a function of the saturation of the vertical wavenumber spectrum. Mcl ntyre cal culates how the constant varies with the saturation of the spectrum. The question is how can the amount of saturation of the spectrum be determined from the measurements?

The parametric models independently estimate the driving noise variance of the input process. Hence, it seems reasonable that the amount of saturation, or gain, of the spectrum relates to the ratio of the input driving noise variance to the average power of the output power spectral density. When the input driving noise is less than the average power, the spectrum is not saturated; when the input driving noise exceeds the average power, the output is saturated with respect to the input. Using the vertical wave number spectra and Mcl ntyre's calculations, a mean value of 0.074 is obtained for $\beta$. 
The resulting eddy diffusion profile is shown in Figure 10. The eddy diffusion coefficient decreases from about $1 \mathrm{~m}^{2} / \mathrm{s}$ at $32 \mathrm{~km}$ to $0.3 \mathrm{~m}^{2} / \mathrm{s}$ around $40 \mathrm{~km}$, before increasing to about $7 \mathrm{~m}^{2} / \mathrm{s}$ at the stratopause.

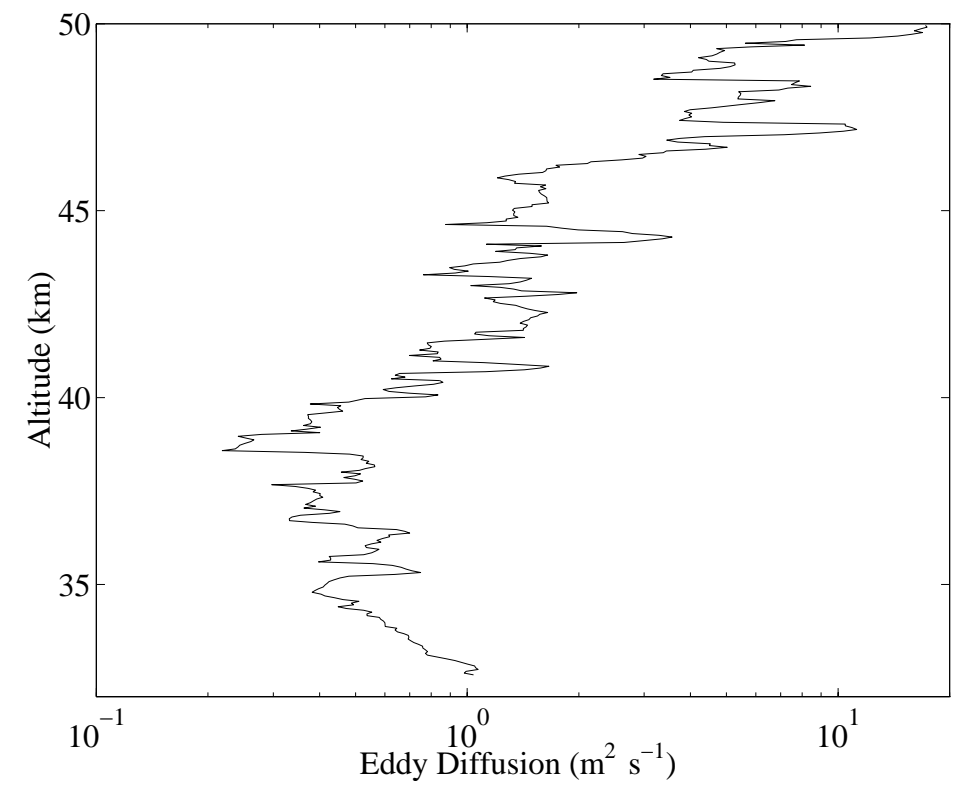

Fig. 10. Eddy diffusion coefficient in the upper stratosphere using the $\beta$ value of Mclntyre (1989) determined from the estimated input driving noise variance of the vertical wavenumber spectrum.

Some variability exists in the determination, in part due to instrumental noise and in part, perhaps in large part, to geophysical variations. The uncertainties are hard to quantify. The absolute value depends on the choice of $\beta$, which varied over time by about $50 \%$. Much of the relative uncertainty is in the calculation of the variance of the accel eration fluctuation which amplifies the high frequency, low power components of the spectrum. Variations also exist in the buoyancy frequency which are greater than the statistical error, and these variations also contribute to the structure evident in the eddy diffusion profile. 


\section{Conclusions}

The measurements of stable and unstable layers has been extended to 13 nights of measurements. The general percentages of layers and layer coherence appear to be similar on nights where the variance of the lapse rates exceed the photocount variance. Maps of the layers on individual nights appear similar (and random) above about $40 \mathrm{~km}$; below these heights the amount of positive and negative layers can show significant structure and variability from night to night. Dr. Hines has suggested that the unstable layers are whitecaps on the crests of large amplitude gravity waves.

The spectral analysis of the Purple Crow Lidar measurements highlight the intermittency of the gravity wave dynamics. Sica (1996b) shows several other examples of the intermittency of the spectra, in addition to mesospheric spectra. Prony's method promises to be useful for exploring the temporal and spatial evolution of individual waves (see Sica (1996c) for details). The energy dissipation, as determined by two independent methods, is larger than suggested in previous studies. The energy dissipation is used to find an eddy diffusion profile. This profile is in reasonable agreement with previous estimates. The estimation of the eddy diffusion profile attempts to include the degree of saturation of the vertical wavenumber spectrum.

Acknowl edgments. RJ S has greatly benefited from scientific discussions with Professors C. Gardner, K. Hamilton, M. Mcl ntyre, T. Shepherd and W. Hocking. Thanks also go to Team Purple Crow, in particular Dr. S. Argall and S. Beatty. This work was supported by grants from the 
National Scientific and Engineering Research Council, the Canadian

Space Agency and Canada's Atmospheric Environment Service.

\section{REFERENCES}

Chanin, M. L. and A. Hauchecorne (1984) Lidar Studies of Temperature and Density Using Rayleigh Scattering, in Handbook for MAP:

Ground-Based Techniques, 13, ICSU Scientific Committee on Solar Terrestrial Physics, Urbana, Illinois, paper 7.

Gardner, C. S., M. S. Miller and C. H. Liu (1989a) Rayleigh lidar observations of gravity wave activity in the upper stratosphere at Urbana, Illinois. J. Atmos. Sci., 46, 1838-1854.

Gardner, C. S., D. C. Senft, T. J . Beatty, R. E. Bills and C. A. Hostetler (1989b) Rayleigh and Sodium Lidar Techniques for Measuring Middle Atmosphere Density, Temperature, and Wind Perturbations and their Spectra. World I onosphere/ Thermosphere Study Handbook, 2, ICSU Scientific Committee on Solar Terrestrial Physics, Urbana, Illinois, Chapter 6.

Hamilton, K. (1984) A study of the occurrence of dynamically unstable conditions in the middle atmosphere, Can. J . Phys., 62, 963-967.

Hines, C. O. (1965) Dynamical heating of the upper atmosphere, J . Geophys. Res., 70, 177-183.

Hines, C. O. (1991) The saturation of gravity waves in the middle atmosphere. Part II: Development of Doppler-spread theory. J . Atmos. Sci., 48, 1360-1379.

Hocking, W. K. (1991) The effects of middle atmosphere turbulence on coupling between atmospheric regions. J. Geomag. Geod ectr., 43, Suppl., 621-636.

Marple, S. L. (1987) Digital spectral analysis. Prentice-Hall, Inc., Chapters 5-11.

Mcl ntyre, ME (1989) On dynamics and transport near the polar mesopause. J . Geophys. Res., 89, 14617-14628. 
Press, W. H., S. A Teukolsky, W. T. Vetterling, and B. P. Flannery (1992) Numerical Recipes in FORTRAN, the Art of Scientific Computing, 2nd edition. Cambridge University Press, 613-622.

Sica, R. J ., S. Sargoytchev, P. S. Argall, E. F. Borra, L. Girard, C. T. Sparrow, and S. Flatt (1995) Lidar measurements taken with a large-aperture liquid mirror. 1. Rayleigh-scatter system. Appl. Opt., 34, 6925-6936.

Sica, R. J . and M. D. Thorsley (1996a) Measurements of superadiabatic lapse rates in the middle atmosphere. Geophys. Res. Lett., submitted.

Sica, R. J . (1996b) Measurements of the Effects of Gravity Waves in the Middle Atmosphere Using Parametric Models of Density Fluctuations. 1: Spatial and Temporal Spectra. J . Atmos. Sci., submitted.

Sica, R. J . (1996c) Measurements of the Effects of Gravity Waves in the Middle Atmosphere Using Parametric Models of Density Fluctuations. 2: Energy Dissipation and Eddy Diffusion. J . Atmos. Sci., submitted. 